\title{
Introduction of the Physiological CTG Interpretation \& Hypoxia in Labour (HIL) Tool, and its Incorporation into a Software Programme: Impact on Perinatal Outcomes
}

\author{
Manjula Samyraju ${ }^{1}$, Sara Ledger ${ }^{2}$ and Edwin Chandraharan ${ }^{3 *}$ \\ ${ }^{1}$ Consultant in obstetrics and Gynecology Safety and intrapartum lead Peterborough, City Hospital North West Anglia, UK \\ ${ }^{2}$ Head of Research \& Development, Baby Lifeline Training Coventry, UK \\ ${ }^{3}$ Director, Global Academy of Medical Education \& Training, London \& Consultant Intrapartum Care Advisor, Mid and South Essex NHS Foundation Trust, \\ Essex, UK
}

Submission: May 17, 2021; Published: June 8, 2021

*Corresponding author: Edwin Chandraharan, Global Academy of Medical Education \& Training, Consultant Intrapartum Care Advisor, UK

\begin{abstract}
Each Baby Counts Reports in the UK from 2016-2021 have consistently highlighted that 33\% of intrapartum related perinatal deaths and severe hypoxic ischaemic injuries in the UK were due to CTG misinterpretation. Moreover, in approximately $72 \%$ of cases, a different care may have given rise to different outcomes. International consensus guidelines on Physiological Interpretation of the CTG produced by 34 CTG experts from 14 countries was aimed at individualisation of care by considering the compensatory response of the individual human fetus to ongoing hypoxic and mechanical stresses during labour and determining the type of ongoing fetal hypoxia. This tool was introduced in a large maternity unit, and it also was incorporated into the Maternity software programme after midwives and obstetricians providing intrapartum care were trained on fetal physiology by conducting Physiological CTG Masterclasses. There was a total elimination of fetal scalp blood sampling (FBS), and a dramatic reduction in the number of babies with severe hypoxic ischaemic encephalopathy.
\end{abstract}

Keywords: Cardiotocograph (CTG); Hypoxic ischaemic encephalopathy (HIE); Physiological CTG interpretation; Hypoxia in labour tool; Fetal monitoring checklist

Abbreviations: CTG: Cardiotocograph; HIE: Hypoxic Ischemic Encephalopathy; NICE: National Institute of Health and Care Excellence; MDT: Multi-Disciplinary Team

\section{Introduction}

The aim of intrapartum fetal heart rate monitoring using the cardiotocograph (CTG) is to avoid intrapartum hypoxia related hypoxic ischemic encephalopathy (HIE) with its long-term sequelae (cerebral palsy and learning difficulties) and perinatal deaths, without increasing unnecessary operative interventions to the mother. This should also be taken alongside the devastation this can cause to women and their families from which some women never recover. The rate of intrapartum emergency interventions (caesarean sections, vacuum, and forceps births) and their resultant complications (postpartum hemorrhage, placenta acreta, 3rd and 4th degree perineal tears) have increased in the United Kingdom (UK), However, there was no concomitant reduction in the rate of intrapartum related hypoxic ischemic brain injuries or perinatal deaths. In fact, Each Baby Counts
Reports which attempted to determine perinatal outcomes for babies born at term in the UK from 2016-2021 have consistently highlighted that $33 \%$ of intrapartum related perinatal deaths and severe hypoxic ischemic injuries in the UK were due to CTG misinterpretation [1]. Moreover, in approximately $72 \%$ of cases, a different care may have given rise to different outcomes [1]. The report by the NHS Resolution on medicolegal payments has also concluded that very little progress about obstetric litigation because of CTG misinterpretation has been made during the last 20 years [2]. A potential drawback of some of the available guidelines for CTG interpretation is that they do not particularly encourage or allow for adjustments for clinical context such as pregnancy complications, gestational age, suspected chorioamnionitis, meconium etc. It is well known that the evidence base for any recommendation regarding fetal monitoring is relatively weak. We 


\section{Global Journal of Reproductive Medicine}

would suggest that the alternative we propose, of recognizing the physiological responses of the individual fetus on the CTG trace, may offer benefits in that delivery is likely to be indicated sooner in the case of significant concerns whilst otherwise reassuring CTG traces with ongoing recurrent variable decelerations would not commit the clinical team to a recommendation of Cesarean delivery. It is not difficult to comprehend that the use of these non-evidencebased, arbitrary parameters on all human fetuses irrespective of the gestational age, individual fetal reserves, and wider clinical picture would increase the risk of poor perinatal outcomes $[3,4]$. In contrast, guideline on Physiological Interpretation of CTG [5] helps determine the physiological compensatory responses of the individual fetus to ongoing intrapartum hypoxic, inflammatory, or mechanical stresses.

\section{What is Physiological Interpretation of CTG?}

Human fetuses are exposed to both hypoxic and mechanical stresses during labor due to repetitive compression of the umbilical cord or episodes of transient reduction in uteroplacental oxygenation because of ongoing uterine contractions. These contractions become progressively stronger, longer, and more frequent as the labor advances. Therefore, like adults being exposed to hypoxic stresses (i.e., running, jogging, exercising in the gym), a fetus is equipped with physiological reflex and endocrine responses to ensure that the fetal central organs (i.e., brain, heart and the adrenals) are protected from hypoxic ischemic injuries during labor. These physiological attempts at compensation to ensure central organ oxygenation can be easily identified on the CTG trace, so that timely action can be taken to avoid hypoxic ischaemic injuries to fetal central organs [6-8]. Features suggestive of rapidly evolving hypoxic stress may require an urgent intervention to improve fetal oxygenation to avoid hypoxic ischemic brain injury [9]. It is also important to recognize that some fetuses may present with evidence of pre-existing hypoxia or injury on the CTG trace [10]. Therefore, they may not be able to withstand additional hypoxic stresses during labour and require an urgent delivery to prevent additional neurological injury. Moreover, some fetuses may experience neurological logical damage non-hypoxic pathways, such as chronic fetal anemia and feto-maternal hemorrhage [11] or intra-amniotic infection resulting in chorioamnionitis [12]. Therefore, continuation of uterine contractions which further reduce oxygenated blood may significantly compromise fetal well-being and potentiate neurological injury in such fetuses with non-hypoxic causes of compromise.

What are the Physiological Tools recommended by the International Consensus Guideline on Physiological Interpretation of CTG?

\begin{tabular}{|l|l|l|l|}
\hline \multicolumn{3}{|c|}{ Table 3 - Checklist to exclude chronic hypoxia and pre-existing fetal injury } \\
\hline 1 & Baseline fetal heart rate appropriate G.A. & Yes & No \\
\hline 2 & Normal variability and cycling & Yes & No \\
\hline 3 & Presence of accelerations (not in labour or latent phase of labour) & Yes & No \\
\hline 4 & No shallow/ late decelerations & Yes & No \\
\hline 5 & $\begin{array}{l}\text { Consider the wider clinical picture: meconium, temperature, fetal growth, reduced fetal } \\
\text { movements }\end{array}$ & Yes & No \\
\hline Overall Impression: Normal / Chronic Hypoxia / Other: & & \\
\hline Management Plan: & \multicolumn{3}{l}{} \\
\hline
\end{tabular}

Figure 1: The Fetal Monitoring Checklist.

The international Consensus Guideline on physiological Interpretation of CTG which has been produced by 34 CTG experts from 14 countries in 2018 [5] has recommended two fetal assessment tools to optimize perinatal outcomes, and to reduce unnecessary intraoperative intervention to the mother. Clinicians need to first determine whether the fetus in question is "FIT to undertake the journey of labor" by applying a "Fetal Monitoring Checklist" (Figure 1) to exclude chronic (i.e., preexisting) hypoxia or evidence of any antenatally fetal compromise (e.g., chorioamnionitis, feto-maternal hemorrhage, chronic fetal anemia, or intrauterine fetal stroke). This should be done at the beginning of CTG recording or at the time of admission. The objective of this approach is very straightforward. Similar to adults who will rapidly become decompensated if the undertake any hypoxic exercise when they are unwell (i.e., due to infection, preexisting cardiac, respiratory or other systemic disorders, severe anemia or hypovolemia and hypotension), fetuses who have sustained an antenatal insult, leading to specific features on the CTG trace indicative of such an ongoing insult, will not be able to sustain further hypoxic stress as a result of ongoing, progressively 


\section{Global Journal of Reproductive Medicine}

worsening and increasing frequency, duration and strength of uterine contractions [12]. A fetus with features on the CTG trace which suggest that "THIS fetus is fit to undertake the journey of labor" [12], like adults, would demonstrate predictable features on the CTG trace, when exposed to intrapartum hypoxic stress $[13,14]$. Therefore, the second, "Intrapartum Fetal Assessment Tool" (Figure 2) is aimed at recognizing the onset of hypoxic and mechanical stresses or fetal inflammation (i.e., chorioamnionitis) by comparing the parameters observed earlier. Therefore, this tool enables individualization of care so that the management can be "tailor-made" to suit the needs of an individual fetus. Moreover, the tool not only helps identify features of the compensation of the central organs, and parameters that might change in nonhypoxic courses of fetal compromise such as fetal heart rate cycling $[11,15,16]$, it also helps to identify the type of fetal hypoxia. Therefore, instead of using arbitrary parameters such as $50 \%$ of the contractions for 30 minutes or 90 minutes, and randomly chosen features in to boxes of "normal", "suspicious" and "pathological", categories with similar interventions to all human fetuses, without determining the fetal response to hypoxic stress and the central organ oxygenation as recommended by the NICE CTG guideline, the International Physiological guideline not only individualized care, but also, helps detect features of non-hypoxic causes of fetal compromise.

\begin{tabular}{|c|c|c|}
\hline \multicolumn{3}{|c|}{ Table 4 - CTG Assessment Tool } \\
\hline bpm Variability & bpm Accelerations & Decelerations \\
\hline Rise in Baseline ( $\geq 10 \%)$ & Yes & No \\
\hline Inter-contraction interval >90 sec & Yes & No \\
\hline Maintained Cycling & Yes & No \\
\hline Abnormal Variability ( $<5$ or $>25$ ) & Yes & No \\
\hline Features of Hypoxia & Yes & No \\
\hline \multicolumn{3}{|l|}{ Type } \\
\hline Central Organs well oxygenated & Yes & No \\
\hline \multicolumn{3}{|l|}{ Other risk factors noted } \\
\hline Recommended Management & & \\
\hline
\end{tabular}

Figure 2: Intrapartum Fetal Assessment Tool.

Our Journey of Implementation of Physiological Interpretation of CTG (Hypoxia in labour tool -HIL tool) at Peterborough City Hospital

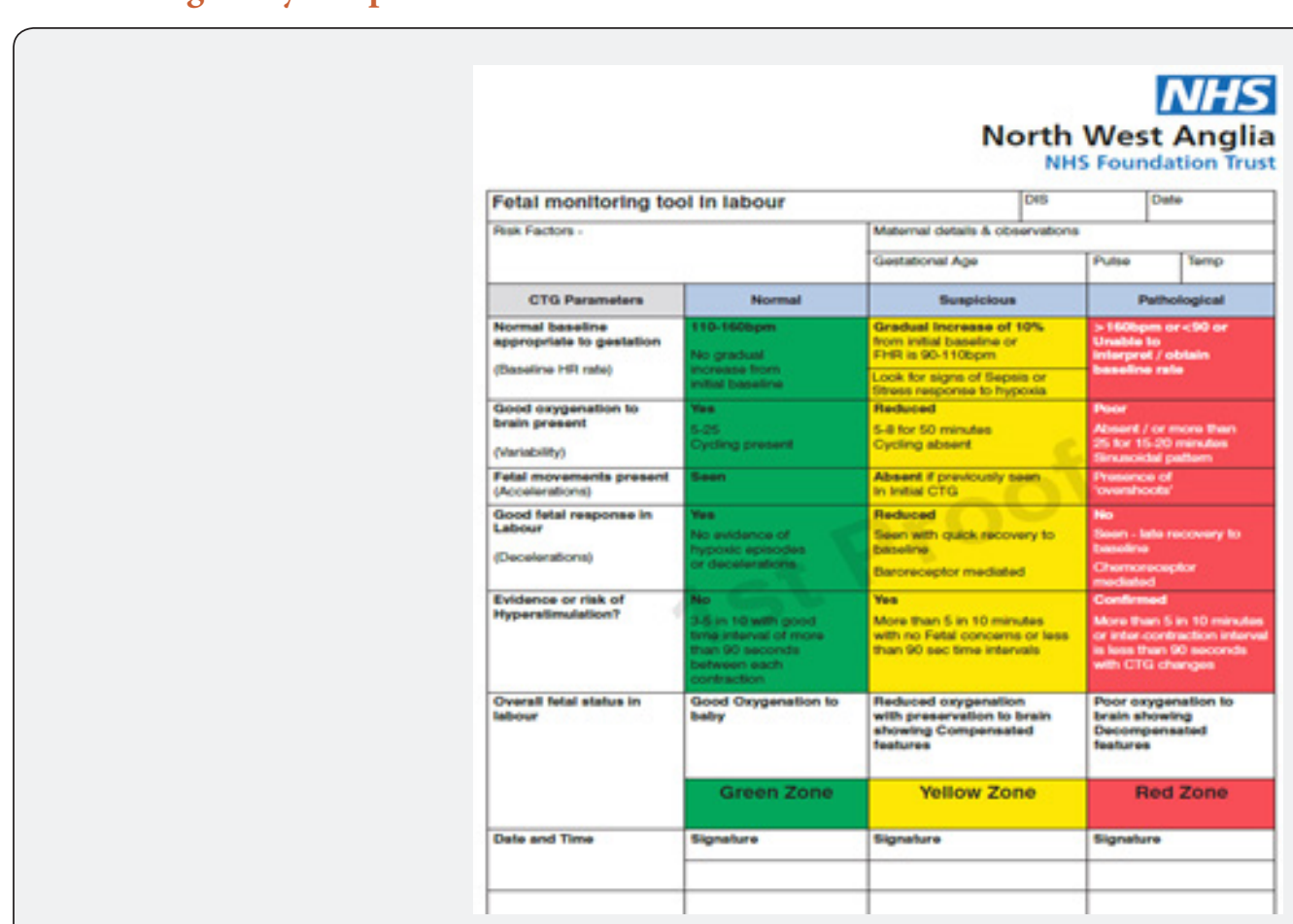

Figure 3: The "hybrid" Intrapartum Fetal Assessment Tool to ensure a smooth transition from FIGO Guidelines to Physiological CTG Interpretation. This tool empowers the clinical team to recognize and escalate to ensure positive outcomes for the mother and baby. 


\section{Global Journal of Reproductive Medicine}

Based on the poor were perinatal outcomes due to the use of NICE CTG Guidelines, are multi-professional team at the Peterborough City Hospital made a collective decision to implement the International CTG Guidelines produced by the International Federation of Gynecology and Obstetrics (FIGO) [17], to reduce confusion and resultant CTG misinterpretation. This was followed by an intense training on Physiological CTG Interpretation to all midwives and obstetricians providing intrapartum care. Unlike some other hospitals who had directly moved from NICE CTG Guidelines to the Physiological CTG Interpretation Tools, we made a collective decision to make a gradual transition. This was mainly because many senior midwives and obstetricians have been used to the CTG classification system based on "Normal", "Suspicious" and "Pathological", for number of years. Therefore, we designed a "hybrid" CTG Assessment Tool (Figure 3) incorporating the principles of Physiological CTG Interpretation as stipulated by the International Physiological CTG Guideline, and the knowledge acquired through Physiological CTG Masterclasses conducted by the Charity Baby Lifeline. We appreciated that it may be argued that the continued use of "Normal", "Suspicious" and "Pathological" categories whilst classifying the CTG traces may increase interobserver variability. However, prior to completely moving to Physiological Interpretation of CTG traces, our senior management team felt that a such a transition was best suited to fulfil the requirements of our local multidisciplinary team. We have attempted to avoid interobserver variability and to optimize intrapartum management by designing another "Management Tool" (Figure 4).

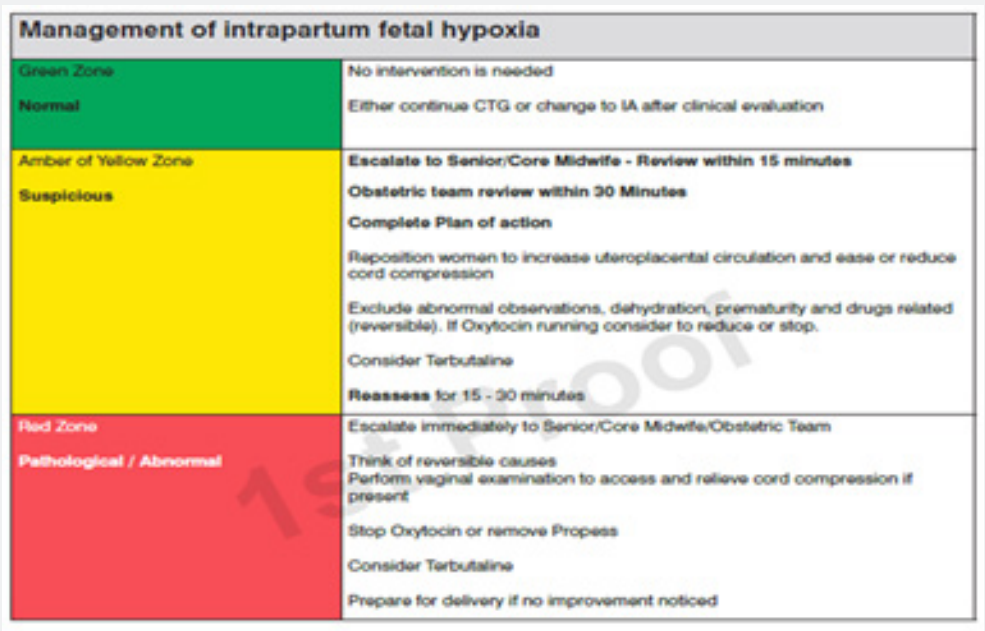

A CTG feature which falls within the Yellow Zone is abnormal and requires a clinical review within 15 - 30 minutes.

A CTG feature which talls within a Red Zone is also abnormal and requires a rapld response.

Figure 4: Intrapartum Management Tool.

What is the Impact of Implementing Physiological Interpretation of Ctg?

Our multidisciplinary team fully embraced the principles of Physiological Interpretation of CTG, and they felt empowered with the knowledge to recognize features of fetal compensation from the onset of fetal decompensation. This was evident almost immediately by complete cessation of the fetal scalp blood sampling (FBS) for "pathological" CTG traces by obstetricians of all grades, from approximately $12-15 \%$ earlier (i.e., 500-700 per year). Cochrane Systematic Reviews had repeatedly concluded that the use of fetal blood sampling (FBS) during labor (both fetal scalp lactate and $\mathrm{pH}$ ) did not appear to influence any long-term neonatal outcomes or reduce intrapartum interventions $[18,19]$, and scientific studies have shown that the use of FBS may in fact significantly increase the rate of emergency caesarean sections $[20,21]$, and its anatomical, physiological, and scientific basis has been repetitively questioned recently [22-25]. Moreover, FBS has been found to be of limited value in the presence of meconium (i.e., bile acids would change the $\mathrm{pH}$ ) [26], and contamination with the amniotic fluid (alkaline pH of the amniotic fluid would neutralize the acidic $\mathrm{pH}$ of the scalp sample leading to a false reassurance) resulting in poor perinatal outcomes [23-25,27]. Rare, but potentially very serious complications such as leakage of cerebrospinal fluid (CSF) as a result of inadvertent incision on the anterior fontanelle have also been reported [28]. Our maternity unit had only used FBS earlier as it was recommended by the NICE Guideline for a "Pathological" CTG trace, due to the lack of understanding of fetal physiological responses to ongoing hypoxic and mechanical stresses. However, as soon as our 
multidisciplinary team understood how a human fetus responds to intrapartum stress, and the features of fetal compensation from decompensation after attending the Physiological CTG Masterclasses, they had immediately stopped performing fetal blood sampling (FBS). This had not only resulted in avoidance of unnecessary trauma and complications to the fetus, and reduction in intrapartum operative interventions to the mother, it had also resulted in financial savings to our hospital because of "FBS kits" not being ordered any longer. Our perinatal outcomes also showed a significant change within two years of implementing Physiological Interpretation of CTG. The number of severe (Grade 2/3) hypoxic ischemic encephalopathy (HIE), which was approximately 10-12 per year prior to conducting Physiological CTG Masterclasses. The number of Grade 2 \& 3 HIE cases in 2014-2015 (2years) was 22. Training on Physiological Interpretation of CTG through CTG Masterclasses were conducted in 2016. There was a progressive reduction in Grade 2 \& 3 HIE cases from 19 in 2016-2017 (2 years) to only 9 in 2018-2020 (3 years). During the last 3 years (20182020 ) we have had only 6 cases (i.e., on average 2 cases per year), which demonstrates $>75 \%$ reduction in the number of Grade $2 \&$ Grade 3 HIE cases after the implementation of Physiological CTG Interpretation, compared to the number of Grade 2 \& 3 HIE in 2015 , This has not only resulted in excellent outcomes and quality of life for babies and their families by reducing the human cost of cerebral palsy, we have ensured a significant financial savings to the NHS. Moreover, the staff morale has also improved by not being involved in serious incidents involving CTG misinterpretation and resultant poor perinatal outcomes. Our multidisciplinary team is very proud of our excellent perinatal outcomes (Figure 5) which has been made possible by intrapartum clinical leadership, multidisciplinary engagement, a drive, and motivation to improve outcomes for women and babies and senior management support.

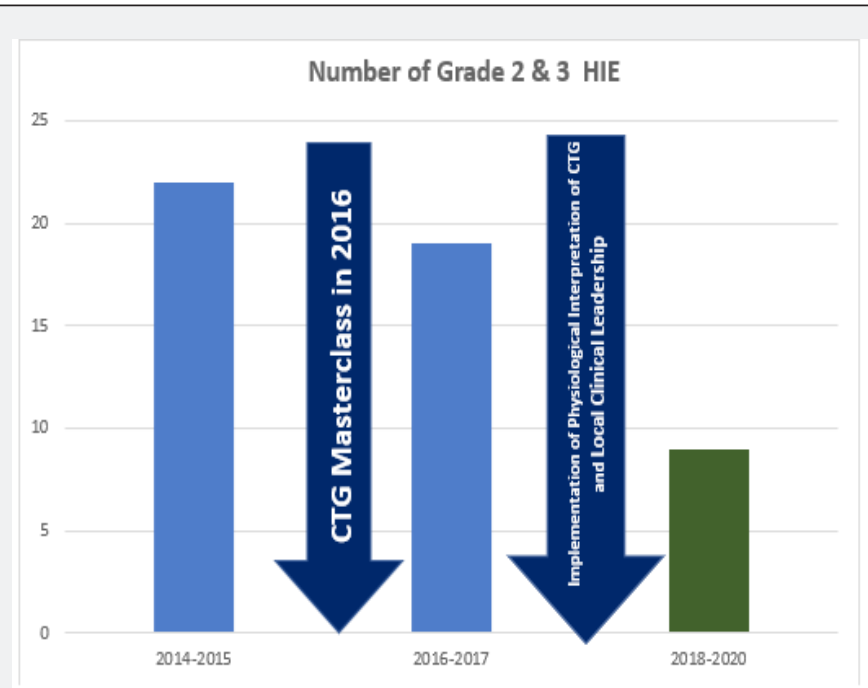

Figure 5: Severe Hypoxic Ischaemic Encephalopathy (HIE) rate before and after the CTG Masterclass (Arrow) and the implementation of change.

Use of Technology to enhance Physiological CTG Interpretation

Our multidisciplinary team embarked on an ambitious project of incorporating the principles of Physiological CTG Interpretation into our existing software program to enable staff to recognize ongoing fetal compromise (Figure 6).

\section{Challenges and Solutions}

Stopping the NICE CTG Guideline which has been traditionally and historically considered as the "gold standard" of intrapartum fetal heart rate monitoring was not easy. There was intense resistance from some senior colleagues and the management. There were concerns regarding our "medicolegal" status if poor outcomes occur with the Physiological Interpretation of CTG traces. Other barriers to change included the human inherent resistance to abandon historical culture and practices, and the lack of "empowerment". Change management requires a unified purpose and an objective, strong strategic leadership, a group of individuals within the organization who are focused on improving outcomes and support from external individuals. Our MDT had a unified purpose and an objective to avoid hypoxic ischemic brain injuries to babies. This objective was shared across the MDT, and with strong clinical leadership, support from external individuals to conduct Physiological CTG Masterclasses to change the mindset, we were able to convince those who were resistant to change. The entire MDT was able to see the poor outcomes because of the continued use of a non-evidence based CTG guideline with predetermined, arbitrarily chosen parameters without individualization of care. They were able to convince those who were worried about "medicolegal" status by highlighting the Bolam Test which is used in clinical negligence 


\section{Global Journal of Reproductive Medicine}

cases. As several other maternity units have also implemented Physiological CTG Guidelines, and the International Consensus Guidelines on Physiological Interpretation of CTG was produced by 34 CTG experts from 14 countries, including several midwives and obstetricians from the UK, we were convinced that our Physiological approach to CTG interpretation would withstand the "Bolam Test" in the Court of law. This is because a responsible body of obstetricians and midwives in the UK currently practice Physiological Interpretation of CTG in their daily, routine clinical practice. Change was difficult, but it was not impossible. It required strong leadership, focus and a desire by the entire team to improve outcomes. Doing the same was not an option because $33 \%$ of babies who had severe hypoxic ischemic brain injury and had died due to intrapartum related causes in the UK were due to CTG misinterpretation. Moreover, according to the Each Baby Counts Reports, approximately $72 \%$ of poor where perinatal outcomes in the UK were potentially avoidable by a different care.
Therefore, our MDT wanted to embrace the difficult process of change, and like other maternity units [29], we have demonstrated improved perinatal outcomes after stopping NICE guidelines and embracing a deeper understanding of physiological responses of a human fetus to ongoing intrapartum hypoxic, inflammatory and mechanical stresses (Figure 7). Understanding of fetal pathophysiological responses and different types of hypoxic would enable midwives and obstetricians to ensure timely interventions to improve outcomes $[8,13,29]$. The historical, illogical practices of fluids to the wrong person (i.e., the mother) to treat "suspicious" CTG traces, and then assessing the $\mathrm{pH}$ of a nonessential fetal scalp tissue for the presence of acidosis (FBS) with the mistaken, nonsensical belief that it would provide information regarding the well-being of fetal central organs, need to change urgently if perinatal outcomes are to be improved in the UK. Staying the same and doing the same about intrapartum fetal heart rate monitoring should no longer be considered as a safe option.

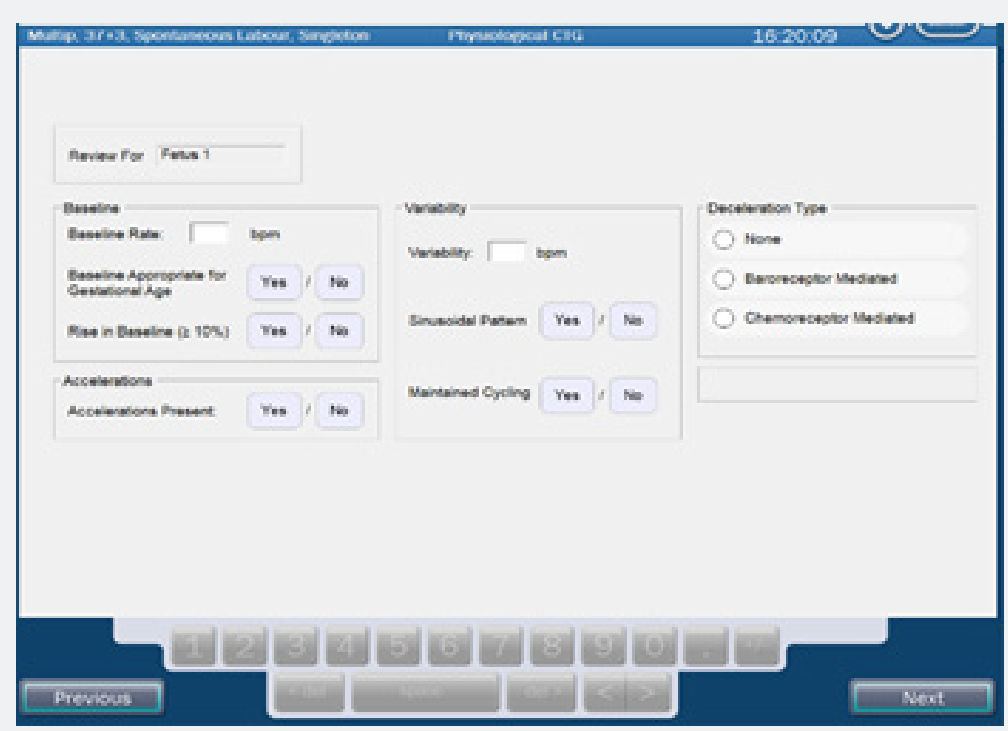

Figure 6: Incorporation of the principles of Physiological Interpretation of CTG into our local CTG software package.

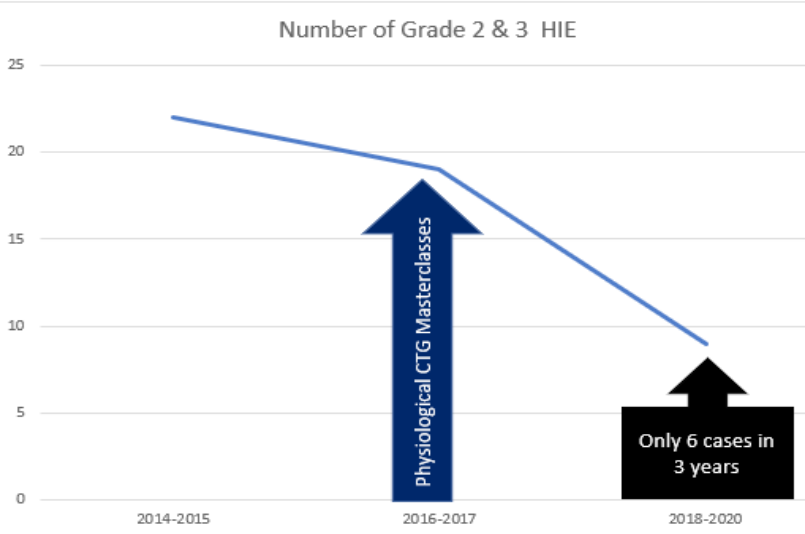

Figure 7: Note the progressive reduction in the number of Grade 2 \& 3 HIE after the implementation of Physiological Interpretation of CTG, Local Clinical Leadership and MDT engagement. 


\section{Global Journal of Reproductive Medicine}

\section{Conclusion}

Our experience from our single center shows that it is possible to change perinatal outcomes by individualization of care by implementing the International Consensus Guidelines on Physiological Interpretation of CTG, and by training midwives and obstetricians through intense Physiological CTG Masterclasses. Our perinatal outcomes demonstrate that the change was not only dramatic, but it was also sustained. We hope that our journey which has eliminated severe hypoxic ischemic brain injury to babies in our maternity unit would motivate and inspire other maternity units to change. Our experience clearly demonstrates predetermined, non-evidence-based, arbitrarily chosen parameters for all human babies without considering the individual fetal reserve, pre-existing compromise and fetal responses to ongoing stress and different types of fetal hypoxia, with Physiological Interpretation of CTG can result in significant improvement in perinatal outcomes. This not only has a significant financial impact to the NHS, but it also avoids the human cost of disability, sadness, and poor quality of life, whilst improving the staff morale. Failure to change for the better in healthcare, when such a change is possible: is this ethically and morally acceptable in contemporary maternity practice?

\section{Contribution to Authorship}

MS and EC conceived the concept and co-wrote the manuscript MS was responsible for collection and analysis of data. All authors reviewed, edited, and approved the final version of the manuscript.

\section{Conflict of Interest}

Ms Manjula Samyraju was the lead clinician for the labour ward who led the implementation of the change, and was instrumental in organizing Physiological CTG Masterclasses, development and implementation of the Tools, local staff support and monitoring outcomes. Ms Sara Ledger is the Head of Training and Research at Baby Lifeline Training and conducts several Physiological CTG Masterclasses to disseminate the knowledge of fetal physiology, and she has provided evidence to a parliamentary select committee to improve maternity training in the UK. Edwin Chandraharan conducts approximately 30 Physiological CTG Masterclasses for about 15 countries. He was the member of the Guideline Development Group for the FIGO CTG Guideline in 2015, and he was on the Editorial Board for the International Consensus Guidelines on Physiological CTG Interpretation with a consensus panel of 34 experts from 14 countries in 2018. He conducted the Physiological CTG Masterclasses at the Peterborough City Hospital via the Charity Baby Lifeline.

\section{References}

1. (2016)Tripling-in-nhs-legal-bills-for-catastrophic-childbirthblunders.

2. Case story Abdominal palpations in labour.
3. (2014) National Institute of Clinical Excellence. Intrapartum care: care of healthy women and their babies during labour. NICE Clinical Guideline.

4. Chandraharan E (2015) Intrapartum care: An urgent need to question historical practices and 'non-evidence'-based, illogical foetal monitoring guidelines to avoid patient harm. Journal of Patient Safety and Risk Management 24(5): 210-217.

5. Chandraharan E, Evans SA, Krueger D, Pereira S, Skivens S, et al. (2018) Physiological CTG interpretation. Intrapartum Fetal Monitoring Guideline.

6. Pinas A, Chandraharan E (2016) Continuous cardiotocography during labour: analysis, classification and management. Best Pract Res Clin Obstet Gynaecol 30: 33-47.

7. Chandraharan E (2017) Handbook of CTG interpretation: from patterns to physiology. (1 $1^{\text {st }}$ Edn.), Cambridge University Press, London.

8. Chandraharan E, Arulkumaran S (2007) Prevention of birth asphyxia: responding appropriately to cardiotocograph (CTG) traces. Best Pract Res Clin Obstet Gynaecol 21: 609-624.

9. Bonfils AGP, Vigneswaran K, Cuadras D, Chandraharan E (2019) Does the saltatory pattern on cardiotocograph (CT G) trace really exist? The ZigZag pattern as an alternative definition and its correlation with perinatal outcomes. J Matern Fetal Neonatal, pp. 1-9.

10. Yanamandra N, Chandraharan E (2013) Saltatory and sinusoidal fetal heart rate (FHR) patterns and significance of FHR overshoots. Curr Women's Health Rev 9: 175-182.

11. Galli L, Dall Asta A, Whelehan V, Archer A, Chandraharan E (2019) Intrapartum cardiotocography patterns observed in suspected clinical and subclinical chorioamnionitis in term fetuses. J Obstet Gynaecol Res 45: 2343-2350.

12. Pereira S, Chandraharan E (2017) Recognition of chronic hypoxia and pre-existing fetal injury on the cardiotocograph (CTG): Urgent need to think beyond the guidelines. Porto Biomed J 2(4): 124-129.

13. Jia YJ, Chen X, Cui HY, Whelehan V, Archer A, et al. (2019) Physiological CTG interpretation: the significance of baseline fetal heart rate changes after the onset of decelerations and associated perinatal outcomes. J Matern Fetal Neonatal Med, pp. 1-6.

14. Oikonomou M, Chandraharan E (2021) Fetal heart rate monitoring in labor: from pattern recognition to fetal physiology. Minerva Obstet Gynecol 73(1): 19-33.

15. Preti M, Chandraharan E (2018) Importance of fetal heart rate cycling during the interpretation of the cardiotocograph (CTG). International J Gynecol Reprod Sci 1: 10-12.

16. Sukumaran S, Pereira V, Mallur S, Chandraharan E (2021) Cardiotocograph (CTG) changes and maternal and neonatal outcomes in chorioamnionitis and/or funisitis confirmed on histopathology. Eur J Obstet Gynecol Reprod Biol 260: 183-188.

17. Ayres-de-Campos D, Spong CY, Chandraharan E (2015) FIGO Intrapartum Fetal Monitoring Expert Consensus Panel. FIGO consensus guidelines on intrapartum fetal monitoring: Cardiotocography. Int J Gynaecol Obstet 131:13-24.

18. Alfirevic Z, Devane D, Gyte GM, Cuthbert A (2017) Continuous cardiotocography (CTG) as a form of electronic fetal monitoring (EFM) for fetal assessment during labour. Cochrane Database Syst Rev 2: CD 006066 .

19. East CE, Leader LR, Sheehan P, Henshall NE, Colditz PB (2010) Intrapartum fetal scalp lactate sampling for fetal assessment in the presence of a non-reassuring fetal heart rate trace. Cochrane Database Syst Rev CD 006174. 
20. Chandraharan E (2014) Fetal scalp blood sampling during labour: is it a useful diagnostic test or a historical test that no longer has a place in modern clinical obstetrics? BJOG 121: 1056-1060.

21. Holzmann M, Wretler S, Cnattingius S, Nordström L (2015) Neonatal outcome and delivery mode in labors with repetitive fetal scalp blood sampling. Eur J Obstet Gynecol Reprod Biol 184: 97-102.

22. Al Wattar BH, Lakhiani A, Sacco A, Siddharth A, Bain A (2019) AB-FAB study group. Evaluating the value of intrapartum fetal scalp blood sampling to predict adverse neonatal outcomes: A UK multicentre observational study. Eur J Obstet Gynecol Reprod Biol 240: 62-67.

23. Chandraharan E, Wiberg N (2014) Fetal scalp blood sampling during labor: an appraisal of the physiological basis and scientific evidence. Acta Obstet Gynecol Scand 93(6): 544-547.

24. Chandraharan E (2014) Fetal scalp blood sampling during labour: is it a useful diagnostic test or a historical test that no longer has a place in modern clinical obstetrics. BJOG 121: 1056-1066.

25. Chandraharan E (2016) Fetal scalp blood sampling should be abandoned: FOR: FBS does not fulfil the principle of first do no harm. BJOG 123: 1770.
26. Chandraharan E (2016) Should national guidelines continue to recommend fetal scalp blood sampling during labor. J Matern Fetal Neonatal Med 29: 3682-3685.

27. Carbonne B, Cudeville C, Maillard F (2003) Study group on fetal pulse oximetry. Predictive value of pulse oximetry and fetal scalp blood $\mathrm{pH}$ in the case of meconium-stained amniotic fluid. Eur J Obstet Gynecol Reprod Biol 109: 27-32.

28. Losch A, Kainz C, Kohlberger P (2003) Influence on fetal blood pH when adding amniotic fluid: an in vitro model. BJOG 110: 453-456.

29. Schaap TP, Moormann KA, Becker JH, Westerhuis ME, Evers A, et al. (2011) Cerebrospinal fluid leakage, an uncommon complication of fetal blood sampling: a case report and review of the literature. Obstet Gynecol Surv 66: 42-46.

30. Chandraharan E, Lowe V, Ugwumadu A, Arulkumaran S (2013) Impact of Fetal ECG (STA N) and competency-based training on intrapartum interventions and perinatal outcomes at a Teaching Hospital in London: 5 Year Analysis. BJOG 120: 428-429.

Your next Submission with Juniper Publishers
will reach you the below assets
- Quality Editorial service
- Swift Peer Review
- Reprints availability
- E-prints Service
- Manuscript Podcast for convenient understanding
- Global attainment for your research
- Manuscript accessibility in different formats
( Pdf, E-pub, Full Text, Audio)
- Unceasing customer service
Track the below URL for one-step submission
https://juniperpublishers.com/online-submission.php

